\title{
Universal conductance of nanowires near the superconductor-metal quantum transition
}

\author{
Subir Sachdev, ${ }^{1}$ Philipp Werner, ${ }^{2}$ and Matthias Troyer $^{2}$ \\ ${ }^{1}$ Department of Physics, Yale University, P.O. Box 208120, New Haven, CT 06520-8120, USA \\ ${ }^{2}$ Theoretische Physik, Eidgenössische Technische Hochschule, CH-8093 Zürich, Switzerland
}

(Dated: February 16, 2004)

\begin{abstract}
We consider wires near a zero temperature transition between superconducting and metallic states. The critical theory obeys hyperscaling, which leads to a universal frequency, temperature, and length dependence of the conductance; quantum and thermal phase slips are contained within this critical theory. Normal (NN), superconducting (SS) and mixed (SN) leads on the wire determine distinct universality classes. For the SN case, wires near the critical point have a universal d.c. conductance which is independent of the length of the wire at low temperatures.
\end{abstract}

The fluctuations of superconducting order in wires have long been the focus of experimental interest, and recent measurements [1, 2, 3] have extended such observations to the nanoscale. Such wires have a diameter which is of the order of the BCS coherence length or larger, so that there are a large number of transverse channels for electronic conduction and the single electron levels are effectively three-dimensional. However, at low energies the collective fluctuations of the superconducting order are one-dimensional because the diameter of the wire is much smaller than its length, L. Above, and not too far below the bulk superconducting critical temperature $T_{c}$, these measurements have been successfully interpreted using a theory [4, [5, 6] based upon the time-dependent Ginzburg-Landau (TDGL) equation. At very low temperatures $(T)$, there is a crossover from the purely classical thermal fluctuations of TDGL theory, to effects associated with quantum fluctuations of the superconducting order. In particular, as the normal state resistance of the wires is increased, they apparently undergo a superconductor to metal quantum transition. In superconducting wires, there is superflow with infinite conductance as $T \rightarrow 0$. In contrast, metallic wires have a finite conductance, $g$, as $T \rightarrow 0$ which decreases inversely with $L, g=\sigma / L$, where $\sigma$ is the conductivity.

This paper will present new results on the transport properties of wires in the vicinity of the $T=0$ quantum superconductor-metal transition (SMT). We will argue that the conductance has a singular contribution which is a universal function of $L, T$, and the measurement frequency, $\omega$, as specified in Eq. (11) below. At a formal level, this scaling form parallels that proposed earlier for the superconductor-insulator transition (SIT) in $d=2$ spatial dimensions $7,[8]$ in the thermodynamic limit; we contend here that such scaling arguments can be extended to the SMT in $d=1$, and to $L$ finite (even though they cannot be extended to the SIT in $d=1$ ). Furthermore, for $L$ finite, we use the theory of surface critical behavior [9] to demonstrate that the leads connected to the sample determine distinct universality classes of the conductance scaling function: wires with superconducting (SS), nor-

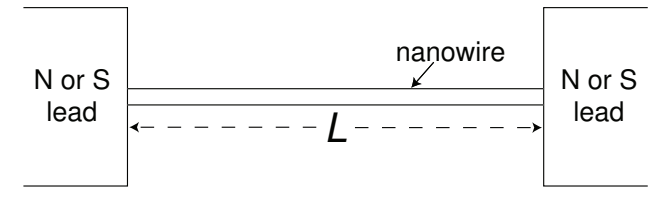

FIG. 1: A wire which is tuned from a superconductor to a metal by (say) reducing its diameter. The leads on the wire are either normal $(\mathrm{N})$ or superconducting $(\mathrm{S})$ and the $\mathrm{NN}$, $\mathrm{SN}$, and SS cases belong to distinct universality classes.

mal (NN), and mixed (SN) leads have distinct scaling functions, but other details of the leads are unimportant (see Fig (1). For wires close to the SMT with $L<L_{T}$, where $L_{T} \sim T^{-1 / z}$ is a thermal cutoff length specified later ( $z$ is the dynamic critical exponent), our arguments imply that as $\omega \rightarrow 0, g=4 e^{2} \mathcal{C}_{X} / h$ (with $X=\mathrm{NN}$, SN, or SS), where the $\mathcal{C}_{X}$ are universal numbers. Thus the d.c. conductance of the wire is independent of $L$ - this happens because the physics is dominated by low energy superconducting fluctuations whose characteristic size is $L$ itself. We will determine the values of $\mathcal{C}_{X}$ in a large $n$ limit ( $n$ is the number of real components of the order parameter, and the case of interest here is $n=2$ ), and by quantum Monte Carlo simulations for a non-random universality class.

The framework of our analysis is provided by a theory for the $T=0$ SMT proposed by Feigel'man and Larkin [10], and examined in a number of studies [11, 12, 13] in $d=2$. This theory may be viewed as a natural quantum extension of the TDGL - it reduces to the TDGL at high $T$. We suspect it is also the theory of critical fluctuations in the analysis of suppression of the critical temperature in Ref. 14. The key to our conclusions is the demonstration [15] that this theory obeys conventional hyperscaling properties at the $T=0 \mathrm{SMT}$ in $d<2$ in the non-random class. Transport properties in the vicinity of the $d=1$ SMT are controlled entirely by the critical theory, and perturbations from irrelevant operators are not needed. In this respect, the situation is similar to the SIT in $d=2$ 
[8]. It is important to note that scaling forms like Eq. (11) do not apply to the non-random SIT in $d=1$ because this SIT is in the Kosterlitz-Thouless universality class, and the conductivity of the superfluid phase near the SIT is determined by 'irrelevant' phase-slip operators 17, 18].

We now state our central scaling hypothesis for the $d=1$ SMT. The conductance always has a 'background' contribution from a parallel metallic channel, and so we write $g=\sigma_{b} / L+\bar{g}$, where $\sigma_{b}$ is the background conductivity which is not expected to have any important $L$, $T$, or $\omega$ dependence; note that the background contribution to the conductance always falls as $1 / L$, and so can become negligible compared to the singular contribution $\bar{g}$. The latter contribution is universal, and obeys the scaling form

$$
\bar{g}(\omega)=\frac{4 e^{2}}{h} \Phi_{X}\left(c_{1} \hbar \omega L^{z}, c_{1} k_{B} T L^{z}, c_{2} L^{1 / \nu}\left(w_{c}-w\right)\right) .
$$

Here $\Phi_{X}$ is a universal scaling function (note that the overall scale of $\Phi_{X}$ is universal and there is no nonuniversal prefactor), $\nu$ is the correlation length exponent, $w$ is the parameter which tunes the wire (say, its diameter) across the superconductor-metal transition at $w=w_{c}$, and $c_{1,2}$ are (the only) non-universal constants. For $w>w_{c}$ Eq. (11) describes metallic conduction, and for $w<w_{c}$ quantum and thermal phase slips disrupt the superflow; we emphasize that, unlike the KosterlitzThouless SIT theory [17], such phase slips are contained within the critical theory of the SMT. In this first discussion here, we focus on the quantum critical behavior of short wires with $L<L_{T} \sim\left(c_{1} k_{B} T\right)^{-1 / z}$ and $L<\left(c_{2}\left|w-w_{c}\right|\right)^{-\nu}$. In this case we can write Eq. (1I) as

$$
\bar{g}(\omega)=\left(4 e^{2} / h\right) F_{X}(y) \quad ; \quad y \equiv c_{1} \hbar \omega L^{z}
$$

where $F_{X}(y)=\Phi_{X}(y, 0,0)$. Computations of the universal function $F_{X}(y)$ are provided below.

We orient ourselves, and estimate various parameters, by recalling the TDGL approach, following the notation of Ref. 6. The spatial $(x)$ and temporal $(t)$ evolution of the complex superconducting order parameter $\Psi(x, t)$ is determined by the classical equation of motion

$$
\hbar \gamma \partial_{t} \Psi=-\left[a+b|\Psi|^{2}-\delta \partial_{x}^{2}\right] \Psi+f
$$

where $f$ is a Langevin noise term, $a=a_{0}\left(T-T_{c}\right) / T_{c}$, and $a_{0}, b, \delta, \gamma$ are $T$-independent parameters whose values are specified in Ref. 6. The dissipative co-efficient $\gamma$ arises from the decay of Cooper pairs into normal electrons. The considerations of Refs. 10, 11 show how a quantized version of Eq. (3) can also apply near a $T=0$ SMT in systems with an inhomogeneous BCS coupling between the electrons, with a reservoir of normal electrons being provided by regions of the sample with a weaker bare coupling (here, these could be near the wire surface). For a wire in the region $0<x<L$, such a theory is described by the imaginary time $(\tau)$ partition function $\mathcal{Z}=\int \mathcal{D} \Psi(x, \tau) \exp \left(-\mathcal{S}_{\text {bulk }}-\mathcal{S}_{\text {boundary }}\right)$ with

$$
\begin{gathered}
\mathcal{S}_{\text {bulk }}=\frac{A}{\hbar} \int_{0}^{L} d x\left[\int_{0}^{\beta} d \tau\left(\delta\left|\partial_{x} \Psi\right|^{2}+a|\Psi|^{2}+\frac{b}{2}|\Psi|^{4}\right)\right. \\
\left.+\frac{\hbar \gamma}{\beta} \sum_{\omega_{n}}\left|\omega_{n}\right|\left|\Psi\left(x, \omega_{n}\right)\right|^{2}\right], \\
\mathcal{S}_{\text {boundary }}=\int_{0}^{\beta} d \tau\left[C_{\ell}|\Psi(0, \tau)|^{2}+C_{r}|\Psi(L, \tau)|^{2}\right. \\
\left.-\operatorname{Re}\left[H_{\ell} \Psi(0, \tau)\right]-\operatorname{Re}\left[H_{r} \Psi(L, \tau)\right]\right] .
\end{gathered}
$$

Here $A$ is the cross-sectional area of the wire, $\beta=$ $\hbar /\left(k_{B} T\right), \omega_{n}$ is a bosonic Matsubara frequency, and $a$ tunes the system across the SMT, and so $a=a_{0}\left(w_{c}-\right.$ $w) / w_{c}$. As a first guess, we may estimate that the parameters $a_{0}, b, \delta$, and $\gamma$ have the same values as those estimated in Ref. 6 in the dirty limit, although the value of $\gamma$ will decrease at low $T$ due to reduced damping. The presence of disorder in the wire also implies a quenched random $x$ dependence of all the couplings in $\mathcal{S}_{\text {bulk }}$; our quantitative results below are limited to the non-random universality class where such $x$ dependence is neglected. The term $\Psi^{*} \partial_{\tau} \Psi$ is permitted in $\mathcal{S}_{\text {bulk }}$ but its co-efficient is proportional to the degree of particle-hole asymmetry on the scaling of pairing energy, and should be quite small: we defer analysis of its consequences to later work. The terms in $\mathcal{S}_{\text {boundary }}$ reflect the presence of the left/right $(\ell / r)$ leads, with $C$ encoding the boundary conditions on the superconducting order [19]. For a $\mathrm{N}$ lead we have $H=0$ and $C>0$, while a $\mathrm{S}$ lead has $H \neq 0$ because the bulk superconductivity of the lead acts like an boundary ordering field on $\Psi$ in the wire.

We now discuss the properties of the QMT of $\mathcal{S}_{\text {bulk }}$ in the thermodynamic limit. These were described recently by Pankov et al. [15]. The QMT has an upper critical dimension of $d=2$, and universal critical properties were computed in an expansion in $\epsilon=2-d$. This expansion obeys hyperscaling properties to all orders in $(2-d)$, and justifies the scaling assumptions behind Eq. (11). The long-range $1 / \tau^{2}$ interaction between $\Psi$ fluctuations generated by the $|\omega|$ dissipative term is preserved under renormalization, and this leads [15, 16] to the exponent identity $z=2-\eta$, where $\eta$ is the anomalous dimension of $\Psi$ which was computed to be $\eta=(n+2)\left(12-\pi^{2}\right) \epsilon^{2} /\left(4(n+8)^{2}\right)$ to order $\epsilon^{2}$. We computed $\nu$ by similar methods to the same order and obtained

$$
\begin{aligned}
& \nu=\frac{1}{2}+\frac{(n+2)}{4(n+8)} \epsilon+ \\
& \frac{(n+2)\left(n^{2}+\left(38-7 \pi^{2} / 6\right) n+132-19 \pi^{2} / 3\right)}{8(n+8)^{3}} \epsilon^{2} .
\end{aligned}
$$


This computation also provides the mean field estimate $L_{T} \sim \sqrt{\delta /\left(\gamma k_{B} T\right)}$. We have also carried out quantum Monte Carlo simulations on a lattice realization of $\mathcal{S}_{\text {bulk }}$ (described below), following those of the $n=1$ case in Ref. 20. The results for the exponents are similar, with $z=1.97(3), z+\eta=1.985(20)$ and $\nu=0.689(6)$. These are in good agreement with the predictions of the $\epsilon$ expansion which upon evaluation for $n=2, \epsilon=1$ yield $\eta=0.021$ and $\nu=0.663$ (from the series for $\nu$ ) or $\nu=0.701$ (from the series for $1 / \nu$ ).

The influence of $\mathcal{S}_{\text {boundary }}$ can be understood using the theory of surface critical behavior [9]. A N lead corresponds to the 'ordinary transition': $C$ is a relevant perturbation which flows to $C=\infty$ under renormalization, and so we have Dirichlet boundary conditions with $\Psi(0 ; L, \tau)=0$ in the scaling limit. Similarly a S lead corresponds to the 'extraordinary transition' in which the magnitude of the ordering field $|H|$ scales to $\infty$. In both cases, the structure of $\Psi$ correlations near the edge is universal, and independent of the specific values of $H$ and $C$. For the SS case there will be a residual universal dependence on the phase difference $\Delta \Phi \equiv \arg \left(H_{\ell}^{*} H_{r}\right)$; we take $\Delta \Phi=0$ in our computations below.

We have now assembled the tools needed to compute the conductance using the Kubo formula 21]. We define

$$
K\left(\omega_{n}\right)=\int_{0}^{\beta} d \tau\langle J(\tau) J(0)\rangle e^{i \omega_{n} \tau}-D
$$

where $J(\tau)=(\delta A /(\hbar L i)) \int_{0}^{L} d x\left(\Psi^{*} \partial_{x} \Psi-\partial_{x} \Psi^{*} \Psi\right)$ and $D=\left(2 \delta A /\left(\hbar L^{2}\right)\right) \int_{0}^{L} d x\left\langle|\Psi|^{2}\right\rangle$. Then $\bar{g}(\omega)=$ $-\left(4 e^{2} / h\right)(2 \pi i / \omega) K\left(\omega_{n} \rightarrow-i \omega\right)$.

A first computation of the conductance was obtained in the $n=\infty$ limit. The bulk theory has the exponents $z=2, \eta=0, \nu=1$. The saddle point equations 22, 23] for finite $L$ involve determination of the optimum $x$-dependent values of the decoupling field for the quartic term at the bulk quantum critical point, and this was done numerically after discretizing $x$ to a lattice. The saddle point solution was inserted into Eq. (7), and leads to the results shown in Fig 2 Note the remarkable insensitivity of the conductance to large changes in the values of the boundary couplings, which confirms our analysis of the surface critical behavior for large $n$. Universality is also demonstrated in Fig [3 where distinct couplings scale to the same conductance in the large $L$ limit.

These results show interesting differences in the small $\omega$ behavior of the conductivity for the NN, SN and SS cases. In the NN case we find $\operatorname{Re}\left[F_{\mathrm{NN}}(y)\right]=\kappa_{1} y^{2}+\ldots$ for small $y$ (the $\kappa$ co-efficients here and below are universal numbers), and hence the universal d.c. conductance vanishes with $\mathcal{C}_{\mathrm{NN}}=0$. This is a consequence of the limited density of states for decay of Cooper pairs at low energies, and we expect this feature to hold beyond the large $n$ expansion (it is also consistent with our Monte
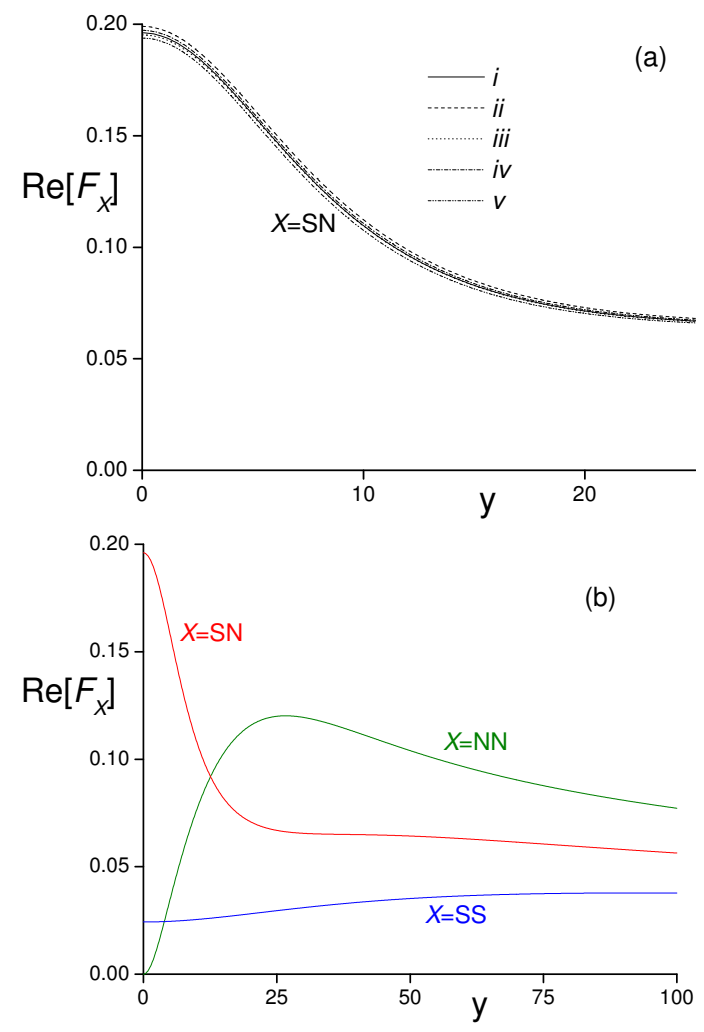

FIG. 2: The universal function $\operatorname{Re}\left[F_{X}\right]$ in the large $n$ limit for real frequencies, with $y=\gamma \hbar \omega L^{2} / \delta$. We discretized $x$ in Eq. (7) on a lattice of spacing unity, and rescaled $\tau$ and $\Psi$ to obtain $\delta A / \hbar=1$ and $A \gamma=1$, and set $b=1$ and used a ultraviolet frequency cutoff $\pi$. (a) Test of universality for the SN case with $L=400$. The parameters $\left(C_{\ell}, C_{r}, H_{\ell}, H_{r}\right)$ are $(i)(1,1,1,0),($ ii $)(1,1,10,0),($ iii $)(1,10,1,0),(i v)(5,1,5,0)$, (v) $(1,1,0.5,0)$. A similar insensitivity to boundary parameters was found for the NN and SN cases. (b) Results for all 3 universality classes are obtained for $L=800$, other parameters as in Fig [2], and $\left(C_{\ell}, C_{r}, H_{\ell}, H_{r}\right)$ taking values $(1,1,0,0)$ for NN, $(1,1,1,0)$ for $\mathrm{SN}$, and $(1,1,1,1)$ for SS. For the SS case, there is an additional Josephson current contribution $F_{\mathrm{SS}}(y)=\pi \varrho \delta(y)$, with $\varrho=2.98$, which is not shown above. All three classes have the common behavior $F_{X}(y \rightarrow \infty)=0.81994(1+i) / \sqrt{y}$ for large $n$.

Carlo results below). In contrast, for the $\mathrm{SN}$ case we find $\operatorname{Re}\left[F_{\mathrm{SN}}(y)\right]=\mathcal{C}_{\mathrm{SN}}+\kappa_{2} y^{2}+\ldots$, with $\mathcal{C}_{\mathrm{SN}}$ non-zero and universal. Here the proximity effect of the $\mathrm{S}$ lead provides a new channel for metallic conduction. Finally, for the SS case we obtain $\operatorname{Re}\left[F_{\mathrm{SS}}(y)\right]=\pi \varrho \delta(y)+\mathcal{C}_{\mathrm{SS}}+\kappa_{3} y^{2}+\ldots$ Here there is a residual Josephson coupling, proportional to the universal number $\varrho$, between the S leads, induced by the proximity effect on both leads.

Finally, we describe our quantum Monte Carlo simulations on a "hard spin" lattice realization of $\mathcal{Z}$. We discretize space into points $j=1 \ldots L$ (integer), imaginary time into points $k=1 \ldots \beta$ (integer measuring $\left.\hbar /\left(k_{B} T\right)\right)$, and set $\Psi\left(x_{j}, \tau_{k}\right)=e^{i \phi_{j, k}}$ to a unit modulus complex number. The continuum theory $\mathcal{Z}$ is realized by 


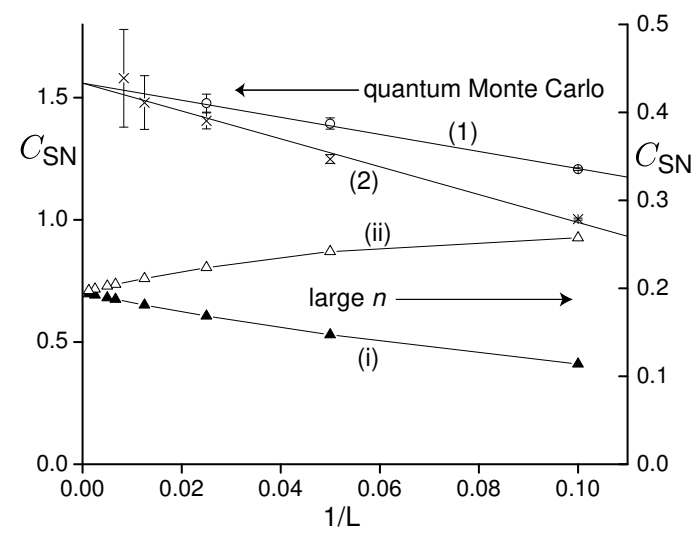

FIG. 3: Extrapolation of the d.c. conductance $\mathcal{C}_{\text {SN }}$ to the universal scaling limit $(L \rightarrow \infty)$. The large $n$ parameters are as in Fig [2] but with $\left(b, C_{\ell}, C_{r}, H_{\ell}, H_{r}\right)=(i)(46.25,1,1,0.745,0)$, (ii) $(0.925,1,1,7.45,0)$. The quantum Monte Carlo parameters are as in the text, with $\left(H_{\ell}, H_{r}\right)=(1)(10,0),(2)(1,0)$.

$$
\begin{aligned}
\mathcal{Z}_{\phi}=\prod_{j=1}^{L} & \prod_{k=1}^{\beta} \int_{0}^{2 \pi} d \phi_{j, k} \exp \left(-\mathcal{S}_{\phi}\right) \text { with } \\
\mathcal{S}_{\phi} & =-K_{x} \sum_{j=1}^{L-1} \sum_{k=1}^{\beta} \cos \left(\phi_{j, k}-\phi_{j+1, k}\right) \\
& -K_{\tau} \sum_{j=1}^{L} \sum_{k=1}^{\beta} \cos \left(\phi_{j, k}-\phi_{j, k+1}\right) \\
& -\sum_{k=1}^{\beta}\left[H_{\ell} \cos \left(\phi_{1, k}\right)+H_{r} \cos \left(\phi_{L, k}\right)\right] \\
+ & \frac{\alpha}{2}\left(\frac{\pi}{\beta}\right)^{2} \sum_{j=1}^{L} \sum_{k=1}^{\beta} \sum_{k^{\prime}=1}^{\beta} \frac{\left[1-\cos \left(\phi_{j, k}-\phi_{j, k^{\prime}}\right)\right]}{\left.\sin ^{2}\left[\pi\left(\tau_{k}-\tau_{k^{\prime}}\right) / \beta\right)\right]}
\end{aligned}
$$

where temporal periodic boundary conditions are imposed by identifying $\phi_{j, \beta+1} \equiv \phi_{j, 1}$. The $K_{\tau}$ term becomes $\left|\partial_{\tau} \Psi\right|^{2}$ in the continuum limit: such a term is formally irrelevant and so was not explicitly noted in $\mathcal{Z}$. Note that the $\alpha$ damping term in $\mathcal{S}_{\phi}$ derives from the $\gamma$ term in $\mathcal{S}_{\text {bulk }}$, and it differs from that usually assumed in the phenomenological resistively-shuntedJosephson (RSJ) junction models [24, 25] - it depends only on cosines of phase differences, while that in the RSJ models depends upon squares of phase differences; this feature is crucial to our results. We chose $\alpha=0.3$, $K_{\tau}=0.1$, and determined the bulk critical point to be at $K_{x}=0.92132(2)$. These values were used in the subsequent computation of the conductance from Eq. (7) with $J\left(\tau_{k}\right)=\left(K_{x} / L\right) \sum_{j=1}^{L-1} \sin \left(\phi_{j, k}-\phi_{j+1, k}\right)$ and $D=\left(K_{x} / L^{2}\right) \sum_{j=1}^{L-1}\left\langle\cos \left(\phi_{j, k}-\phi_{j+1, k}\right)\right\rangle$.

We obtained Monte Carlo results for $F_{X}(y)$ along the imaginary frequency axis, and the results had a structure similar to those of the large $n$ theory. We show in Fig 3 the values of the universal d.c. conductance $\mathcal{C}_{S N}$ as a function of $1 / L$ : the large $n$ theory is seen to significantly underestimate its value, but has a similar sensitivity to finite sizes.

This paper has described the consequences of a theory [10, 11, 12, 13] for a quantum transition between a superconductor and a metal in one spatial dimension. Our results apply to wires in which the superconducting 'Cooperon' fluctuations are effectively one-dimensional, but there are a very large number of transverse singleelectron channels so that the strictly one-dimensional Luttinger liquid physics does not apply. We have argued that the proliferation of thermal and quantum phase slips near such a transition is conveniently described by a 'softspin' continuum theory in Eqs. (45), whose critical point obeys conventional hyperscaling properties. We used analytic and Monte Carlo computations to make predictions on a universal conductance. We hope that future experiments on short wires can test our predictions, and particularly their sensitivity to the leads; other recent works [25, 26] have also addressed lead-sensitivity, but in very different frameworks.

We thank A. Bezryadin, J. Cardy, S. Chakravarty, D. Dalidovich, E. Demler, M. Devoret, A. Finkel'stein, M. P. A. Fisher, S. Girvin, P. Phillips, G. Refael, B. Spivak, and A. D. Stone for valuable discussions. This research was supported by the National Science Foundation under grant DMR-0098226 (S.S.) and by the Swiss National Science Foundation (M.T.). The calculations have been performed on the Asgard Beowulf cluster at ETH Zürich, using the parallellizing Monte Carlo library of the open source ALPS project [27].

[1] A. Bezryadin, C. N. Lau, and M. Tinkham, Nature (London) 404, 971 (2000); A. Rogachev and A. Bezryadin, Appl. Phys. Lett. 83, 512 (2003); A. T. Bollinger, A. Rogachev, M. Remeika, and A. Bezryadin, cond-mat/0401350

[2] C. N. Lau, N. Markovic, M. Bockrath, A. Bezryadin, and M. Tinkham, Phys. Rev. Lett. 87, 217003 (2001).

[3] G. R. Boogaard, A. H. Verbruggen, W. Belzing, and T. M. Klapwijk, cond-mat/0401364

[4] J. S. Langer and V. Ambegaokar, Phys. Rev. 164, 498 (1967).

[5] D. E. McCumber and B. I. Halperin, Phys. Rev. B 1, 1054 (1970).

[6] J. R. Tucker and B. I. Halperin, Phys. Rev. B 3, 3768 (1971).

[7] M. P. A. Fisher, G. Grinstein, and S. M. Girvin, Phys. Rev. Lett. 64, 587 (1990).

[8] K. Damle and S. Sachdev, Phys. Rev. B 56, 8714 (1997).

[9] H. W. Diehl in Phase Transitions and Critical Phenomena, v. 10, C. Domb and J. Lebowitz eds., Academic Press, London (1986).

[10] M. V. Feigel'man and A. I. Larkin, Chem. Phys. 235, 107 (1998); M. V. Feigel'man, A. I. Larkin, and M. A. Skvortsov, Phys. Rev. Lett. 86, 1869 (2001).

[11] B. Spivak, A. Zyuzin, and M. Hruska, Phys. Rev. B 64, 132502 (2001).

[12] D. Dalidovich and P. Phillips, Phys. Rev. Lett. 84, 737 (2000); Phys. Rev. B 66, 073308 (2002); cond-mat/0310129 
[13] I. F. Herbut, Phys. Rev. Lett. 85, 1532 (2000).

[14] Y. Oreg and A. M. Finkel'stein, Phys. Rev. Lett. 83, 191 (1999).

[15] S. Pankov, S. Florens, A. Georges, G. Kotliar, and S. Sachdev, Phys. Rev. B in press, cond-mat/0304415

[16] A. Gamba, M. Grilli, and C. Castellani, Nucl. Phys. B 556, 463 (1999).

[17] T. Giamarchi, Phys. Rev. B 46, 342 (1992).

[18] A. D. Zaikin, D. S. Golubev, A. van Otterlo, and G. T. Zimányi, Phys. Rev. Lett. 78, 1552 (1997); D. S. Golubev and A. D. Zaikin, Phys. Rev B 64, 014504 (2001). These authors claim a theory for the $d=1$ SMT, but we maintain their theory is actually for the $d=1$ SIT. Their results agree with those of the $d=1$ SIT obtained by Giamarchi [17], where they overlap.

[19] P. G. de Gennes, Superconductivity of Metals and Alloys, W. A. Benjamin, New York (1966), section 7-3.
[20] P. Werner, K. Völker, M. Troyer, and S. Chakravarty, cond-mat/0402224

[21] D. S. Fisher and P. A. Lee, Phys. Rev. B 23, 6851 (1981).

[22] S. Sachdev, A. V. Chubukov, and A. Sokol, Phys. Rev. B 51, 14874 (1995).

[23] A. J. Bray and M. A. Moore, J. Phys. A 10, 1927 (1977).

[24] S. Chakravarty, G. Ingold, S. Kivelson, and G. Zimányi, Phys. Rev. B 37, 3283 (1988).

[25] G. Refael, E. Demler, Y. Oreg, and D. S. Fisher, in preparation; G. Refael, Ph.D. thesis, Harvard University (2003).

[26] H. P. Büchler, V. B. Geshkenbein, and G. Blatter, Phys. Rev. Lett. 92, 067007 (2004).

[27] M. Troyer et al., Lec. Notes in Comp. Sci. 1505, 191 (1998). Source codes of the libraries are available from http://alps.comp-phys.org/. 